This item was submitted to Loughborough's Research Repository by the author.

Items in Figshare are protected by copyright, with all rights reserved, unless otherwise indicated.

\title{
Scanning LDV using wedge prisms
}

PLEASE CITE THE PUBLISHED VERSION

http://dx.doi.org/10.1016/j.optlaseng.2008.06.003

PUBLISHER

(c) Elsevier Ltd.

VERSION

AM (Accepted Manuscript)

LICENCE

CC BY-NC-ND 4.0

REPOSITORY RECORD

Tirabassi, Mario, and Steve Rothberg. 2019. "Scanning LDV Using Wedge Prisms". figshare. https://hdl.handle.net/2134/8842. 
This item was submitted to Loughborough's Institutional Repository (https://dspace.lboro.ac.uk/) by the author and is made available under the following Creative Commons Licence conditions.

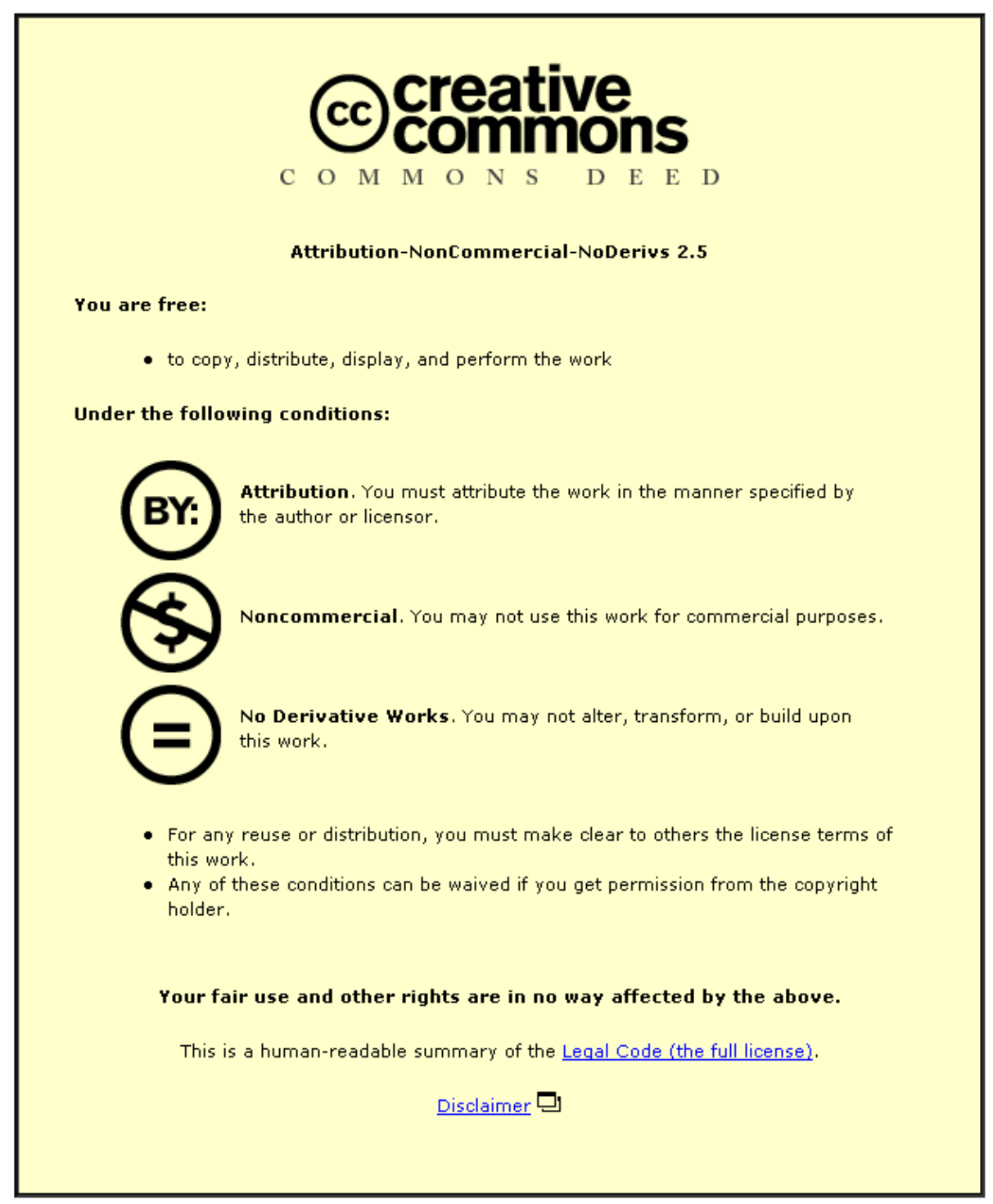

For the full text of this licence, please go to: http://creativecommons.org/licenses/by-nc-nd/2.5/ 


\title{
Scanning LDV using wedge prisms
}

\author{
Mario Tirabassi and Steve J. Rothberg \\ Mechanical and Manufacturing Engineering, Loughborough University, \\ Loughborough, Leics, LE11 3TU, UK \\ M.Tirabassi@lboro.ac.uk, \\ S.J.Rothberg@lboro.ac.uk
}

\section{Abstract}

Recent work on Laser Vibrometry proposed a mathematical model for the velocity sensed by a laser beam incident in an arbitrary direction on a rotating target undergoing arbitrary vibration. A single arbitrary point along the line of incidence of the laser beam must be known, together with an arbitrary known point along the line of the beam and the main purpose of this paper is to introduce a general procedure to determine both. This new procedure is applied to a novel, scanning LDV arrangement in which rotating wedge prisms are used in order to track a rotating component. Special attention is given to the time-dependent laser beam orientation using a vector description of refraction to enable concise expression. Experimental data is presented for the first time suggesting advantages over the convention dual mirror system currently used for tracking LDV.

Keywords: laser Doppler vibrometry (LDV), scanning, tracking, velocity sensitivity, rotating machinery, vibration measurement. 


\section{1 - Introduction}

The principle of Laser Doppler Vibrometry (LDV) is in the detection of a Doppler shift in the frequency of coherent light scattered by a moving target, from which a time resolved measurement of the target velocity is obtained. The non-contact Laser Vibrometer is now well established as an effective alternative to the traditional contacting vibration transducer. In rotating machinery design and development applications, where assessment of vibration is essential, important measurements using LDV [1] and scanning LDV [2] have been performed.

Commercial scanning Vibrometers incorporate two orthogonally aligned mirrors to deflect the laser beam and can operate point by point or in a continuous scanning mode [3] such as the tracking mode [4]. In the circular tracking mode, the mirrors must oscillate continuously and operation becomes difficult at high rotational speeds because of the inertia of the scanning mirrors. Alternative solutions requiring whole body rotation of optical devices rather than oscillation include the use of a derotator [5], or replacement of the electro-mechanical connection between the structure and tracking system with a complete mechanical connection $[6,7]$. These devices deflect the laser beam through reflection and/or refraction, emphasising the value of a versatile method of predicting incident beam orientation such as that proposed in this paper. The method is applied to a novel arrangement in which two rotating wedges enable the tracking of a rotating component. (The circular scan is possible with a single wedge but use of two wedges provides flexibility in scan diameter). A further limitation of the conventional dual mirror 
scanning configuration is the presence of a measured velocity component at twice the scan frequency in tracking measurements on rotating components, even for zero misalignment and without target vibration [8]. For the new arrangement, this paper will explore any similar effects.

\section{2 - Velocity sensitivity model for the wedge scheme}

\section{1 - The general velocity sensitivity model: review}

The general velocity sensitivity model [9] asserts that the velocity measured by a single incident laser beam is:

$U_{m}=\hat{b} \cdot \vec{V}_{P}$

where $\hat{b}$ is the unit vector for the incident beam direction and $\vec{V}_{P}$ is the total velocity of the incident point $P$. This paper presents a general procedure to determine the vector $\hat{b}$.

For scanning, laser beam deflection is possible using mirrors or prisms positioned along the optical path. When a laser beam is incident at an interface between homogeneous media with different refractive indices, $\varepsilon_{t}$ and $\varepsilon_{i}$, as shown in figure 1 , reflection and refraction can occur. The vector expression for the reflected beam is given by [8]: 
$\hat{b}_{r}=\hat{b}_{i}-2\left(\hat{b}_{i} \cdot \hat{n}\right) \hat{n}$

and that for the refracted beam is [10]:

$\hat{b}_{t}=\left(\hat{b}_{i}-\left(\hat{b}_{i} \cdot \hat{n}\right) \hat{n}\right) \frac{\varepsilon_{i}}{\varepsilon_{t}}+\left(\sqrt{1-\left(\frac{\varepsilon_{i}}{\varepsilon_{t}}\right)^{2}\left(1-\left(\hat{b}_{i} \cdot \hat{n}\right)^{2}\right)}\right) \hat{n}$

where $\hat{b}_{i}, \hat{b}_{r}$ and $\hat{b}_{t}$ are, respectively, the unit vectors for the incident, reflected and refracted beams and $\hat{n}$ is the normal to the interface between the two surfaces. Equations (2) and (3) show that knowledge of the incident beam direction and the surface normal lead to outgoing beam direction for both reflection and refraction.

\subsection{Beam orientation for scanning with rotating wedges}

The general procedure to determine the final beam orientation will be applied to the optical arrangement shown in figure 2. The reference system $x y z$ maintains its direction at all times, the $z$ axis coincides with the undeflected target spin axis and the origin $O$ is fixed to a point on the $z$ axis. Two rotating wedges, the first rotating around the axis $B C$ and the second around the axis $F G$, are positioned as shown in figure 2. When the wedge rotations are synchronized with the rotation of the target, the arrangement enables circular tracking. The final beam orientation is obtained by applying equation (3) at each refraction and this will now be demonstrated for the case of zero misalignment i.e. the incident laser beam, the wedge rotation axes and the target spin axes are collinear with the $z$ axis. 
The laser beam incident on the vertical surface of the first wedge is described by the unit vector $\hat{b}_{1}$ :

$$
\hat{b}_{1}=\left[\begin{array}{lll}
\hat{x} & \hat{y} & \hat{z}
\end{array}\right]\left[\begin{array}{lll}
0 & 0 & -1
\end{array}\right]^{T}
$$

On any wedge surface, all points have the same normal direction. Using rotation matrices, see appendix 1 for further details, the two surface normal directions for the first wedge are given as:

$\hat{u}_{B}=\left[\begin{array}{lll}\hat{x} & \hat{y} & \hat{z}\end{array}\right]\left[\begin{array}{lll}0 & 0 & -1\end{array}\right]^{T}$

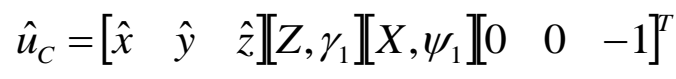

The matrix $\left[Z, \gamma_{1}\right]$ represents the whole body rotation of the wedge around its spin axis, with the rotation angle $\gamma_{1}=\Omega_{1} t+\phi_{1}$ in which $\Omega_{1}$ is the rotational speed of the wedge (equal to the target rotational speed to perform tracking) and the phase $\phi_{1}$ denotes the initial angular position of the wedge. The matrix $\left[X, \psi_{1}\right]$ incorporates the inclination $\psi_{1}$ of the wedge. In the same way, the surface normals for the second wedge are given as:

$$
\begin{aligned}
& \hat{u}_{H}=\hat{u}_{F}=\left[\begin{array}{lll}
\hat{x} & \hat{y} & \hat{z} \llbracket\left[Z, \gamma_{2} \llbracket\left[X, \psi_{2}\right.\right.
\end{array}\right]\left[\begin{array}{lll}
0 & 0 & -1
\end{array}\right]^{T} \\
& \hat{u}_{J}=\hat{u}_{G}=\left[\begin{array}{llll}
\hat{x} & \hat{y} & \hat{z}
\end{array} \llbracket\left[\begin{array}{lll}
0 & 0 & -1
\end{array}\right]^{T}\right.
\end{aligned}
$$

where $\psi_{2}$ is the inclination of the second wedge and $\gamma_{2}$ is its rotational angle 
around the $z$ axis defined as $\gamma_{2}=\Omega_{2} t+\phi_{2}$, in which $\Omega_{2}$ is the rotational speed of the wedge and $\phi_{2}$ denotes its initial angular position. The phases $\phi_{1}$ and $\phi_{2}$ control the scan radius.

To define the final beam orientation, it is necessary to consider the change in the beam orientation at each interface. For zero misalignment, $\hat{b}_{1}$ is perpendicular to the first surface of the first wedge and no refraction takes place, thus $\hat{b}_{2}=\hat{b}_{1}$. The same result is obtained by applying equation (3) at the point $B$ :

$\hat{b}_{2}=\left(\hat{b}_{1}-\left(\hat{b}_{1} \cdot \hat{u}_{B}\right) \hat{u}_{B}\right) \frac{\varepsilon_{a}}{\varepsilon_{w l}}+\left(\sqrt{1-\left(\frac{\varepsilon_{a}}{\varepsilon_{w l}}\right)^{2}\left(1-\left(\hat{b}_{1} \cdot \hat{u}_{B}\right)^{2}\right)}\right) \hat{u}_{B}$

where $\varepsilon_{w l}$ and $\varepsilon_{a}$ are, respectively, the refractive indices of the first wedge and air. Then, the beam is refracted at point $C$ and the orientation of $\hat{b}_{3}$ is described by:

$\hat{b}_{3}=\left(\hat{b}_{2}-\left(\hat{b}_{2} \cdot \hat{u}_{C}\right) \hat{u}_{C}\right) \frac{\varepsilon_{w 1}}{\varepsilon_{a}}+\left(\sqrt{1-\left(\frac{\varepsilon_{w l}}{\varepsilon_{a}}\right)^{2}\left(1-\left(\hat{b}_{2} \cdot \hat{u}_{C}\right)^{2}\right)}\right) \hat{u}_{C}$

On the second wedge, the refractions take place at the point $H$ and $J$ and the beam orientations are defined by the following equations:

$$
\begin{aligned}
& \hat{b}_{4}=\left(\hat{b}_{3}-\left(\hat{b}_{3} \cdot \hat{u}_{F}\right) \hat{u}_{F}\right) \frac{\varepsilon_{a}}{\varepsilon_{w 2}}+\left(\sqrt{1-\left(\frac{\varepsilon_{a}}{\varepsilon_{w 2}}\right)^{2}\left(1-\left(\hat{b}_{3} \cdot \hat{u}_{F}\right)^{2}\right)}\right) \hat{u}_{F} \\
& \hat{b}_{5}=\left(\hat{b}_{4}-\left(\hat{b}_{4} \cdot \hat{u}_{G}\right) \hat{u}_{G}\right) \frac{\varepsilon_{w 2}}{\varepsilon_{a}}+\left(\sqrt{1-\left(\frac{\varepsilon_{w 2}}{\varepsilon_{a}}\right)^{2}\left(1-\left(\hat{b}_{4} \cdot \hat{u}_{G}\right)^{2}\right)}\right) \hat{u}_{G}
\end{aligned}
$$


In this way, the procedure allows straightforward description of the final beam orientation, readily incorporating the geometrical complexity in the configuration under consideration.

\subsection{Determination of the 'known' point}

To apply the velocity sensitivity model, the incident beam orientation and knowledge of an arbitrary point along the ultimate line of the incident beam are required. For a single wedge, the final beam orientation is $\hat{b}_{3}$ given by equation (10) and the point $\mathrm{C}$, found from vector $\overrightarrow{O C}$, is convenient to use:

$\overrightarrow{O C}=\overrightarrow{O B}-|\overrightarrow{B C}| \hat{z}$

The incident point in the plane of the target is then given by:

$$
\overrightarrow{O K}=\overrightarrow{O C}+|\overrightarrow{C K}| \hat{b}_{3}=\overrightarrow{O C}+\left[\frac{\overrightarrow{O C} \cdot \hat{z}}{\hat{b}_{3} \cdot \hat{z}}\right] \hat{b}_{3}
$$

For the system with two wedges, the point $J$ from which the final beam $\hat{b}_{5}$

originates is the obvious choice of 'known' point and is obtained using vector polygons, as follows:

$\overrightarrow{O J}=\overrightarrow{O C}+\overrightarrow{C H}+\overrightarrow{H J}$

The point $H$ from which the beam $\hat{b}_{4}$ originates is determined from the following vector system: 
$\left\{\begin{array}{l}\overrightarrow{O F}=\overrightarrow{O C}+\overrightarrow{C H}+\overrightarrow{H F} \\ \overrightarrow{H F} \cdot \hat{u}_{H}=0\end{array}\right.$

which reveals that

$\overrightarrow{C H}=\left[\frac{(\overrightarrow{O F}-\overrightarrow{O C}) \cdot \hat{u}_{H}}{\hat{b}_{3} \cdot \hat{u}_{H}}\right] \hat{b}_{3}$

In the same manner, the position for the point $J$ is obtained from the following system:

$\left\{\begin{array}{l}\overrightarrow{G J}=\overrightarrow{O C}+\overrightarrow{C H}+\overrightarrow{H J}-\overrightarrow{O G} \\ \overrightarrow{O G}=\overrightarrow{O F}-|\overrightarrow{F G}| \hat{z} \\ \overrightarrow{G J} \cdot \hat{u}_{J}=0\end{array}\right.$

from which it can be shown that:

$\overrightarrow{H J}=\left[\frac{(\overrightarrow{O G}-\overrightarrow{O C}-\overrightarrow{C H}) \cdot \hat{u}_{J}}{\hat{b}_{4} \cdot \hat{u}_{J}}\right] \hat{b}_{4}$

The position of $\mathrm{J}$ then follows from substitution of equations (17) and (19) into (15).

The incident point in the plane of the target is then given by:

$\overrightarrow{O K}=\overrightarrow{O J}+|\overrightarrow{J K}| \hat{b}_{5}=\overrightarrow{O J}+\left[\frac{\overrightarrow{O J} \cdot \hat{z}}{\hat{b}_{5} \cdot \hat{z}}\right] \hat{b}_{5}$

2.5 Measured velocity and beam path in a tracking system with a single wedge 
For a scanning system with a single wedge, the final laser beam direction is given by expansion of equation (10) as:

$$
\hat{b}_{3}=\hat{x}\left[Q \sin \psi_{1} \sin \left(\Omega_{1} t+\varphi_{1}\right)\right]-\hat{y}\left[Q \sin \psi_{1} \cos \left(\Omega_{1} t+\varphi_{1}\right)\right]+\hat{z}\left[Q \cos \psi_{1}-\frac{\varepsilon_{w 1}}{\varepsilon_{a}}\right]
$$

where:

$$
Q=\cos \psi_{1} \frac{\varepsilon_{w 1}}{\varepsilon_{a}}-\sqrt{1-\left(\frac{\varepsilon_{w 1}}{\varepsilon_{a}}\right)^{2}\left(\sin ^{2} \psi_{1}\right)}
$$

Equation (21a) shows that the $x$ - and $y$-components of beam orientation are periodic functions of the wedge rotation speed, while the z-component is constant. Substituting equation (21a) into equation (1), the velocity measured by a laser beam incident on a rotating target of flexible cross-section is given by:

$$
\begin{aligned}
U_{m}= & Q \sin \psi_{1} \sin \left(\Omega_{1} t+\phi_{1}\right)\left\lfloor\dot{x}_{r}\left(P_{0}\right)+\dot{x}_{f}(P)\right\rfloor \\
& -Q \sin \psi_{1} \cos \left(\Omega_{1} t+\phi_{1}\right)\left[\dot{y}_{r}\left(P_{0}\right)+\dot{y}_{f}(P)\right] \\
& +\left(Q \cos \psi_{1}-\frac{\varepsilon_{w 1}}{\varepsilon_{a}}\right)\left[\dot{z}_{r}\left(P_{0}\right)+\dot{z}_{f}(P)\right]
\end{aligned}
$$

where $\dot{x}_{f}(P), \dot{y}_{f}(P)$ and $\dot{z}_{f}(P)$ are the vibration velocity components due to the target cross-section flexibility and $\dot{x}_{r}\left(P_{0}\right), \dot{y}_{r}\left(P_{0}\right)$ and $\dot{z}_{r}\left(P_{0}\right)$ are the resultant vibration velocity components due to rigid body vibration [8]:

$$
\begin{aligned}
& \dot{x}_{r}\left(P_{0}\right)=\dot{x}-\left(\dot{\theta}_{z}+\Omega\right)\left(y_{0}-y\right)+\left(\dot{\theta}_{y}-\Omega \theta_{x}\right)\left(z_{0}-z\right) \\
& \dot{y}_{r}\left(P_{0}\right)=\dot{y}+\left(\dot{\theta}_{z}+\Omega\right)\left(x_{0}-x\right)-\left(\dot{\theta}_{x}+\Omega \theta_{y}\right)\left(z_{0}-z\right)
\end{aligned}
$$


$\dot{z}_{r}\left(P_{0}\right)=\dot{z}+\left(\dot{\theta}_{x}+\Omega \theta_{y}\right)\left(y_{0}-y\right)-\left(\dot{\theta}_{y}-\Omega \theta_{x}\right)\left(x_{0}-x\right)$

where $\dot{\theta}_{x}, \dot{\theta}_{y}, \dot{\theta}_{z}$ and $\theta_{x}, \theta_{y}, \theta_{z}$ are the angular vibration velocities and displacements of the target around the $\mathrm{x}, \mathrm{y}, \mathrm{z}$ axes and $\left(x_{0}, y_{0}, z_{0}\right)$ is the position of an arbitrary known point that lies along the line of the beam. The $(x, y, z)$ coordinates of point $\mathrm{C}$ are used in equations (22b-d). The incident beam describes an apparently perfect circular path centred in the $x y$ plane, useful for tracking applications, as shown in the plot of vector $\overrightarrow{O K}$ in figure 3. Substituting equations (22b-d) into equation (22a) and synchronising the rotation speeds of the wedge and the target $\Omega_{I}=\Omega$, the measured velocity per unit rotation speed in the absence of target vibration is given by:

$\frac{U_{m}}{\Omega}=-y_{0} \sin \psi_{1} Q \sin \left(\Omega_{1} t+\varphi_{1}\right)-x_{o} \sin \psi_{l} Q \cos \left(\Omega_{l} t+\varphi_{1}\right)$

This type of analysis has been useful previously to consider any measured velocity components resulting from the whole body target rotation. With point $C,\left(0,0, z_{C}\right)$, as the 'known' point, the velocity sensed by the vibrometer is readily seen to be zero. Of note here is that the single wedge can perform the task of the 2 orthogonal mirrors in a conventional scanning system without introducing the velocity component at $2 x$ scan frequency or the distortion of the circular path that are both inherent in the conventional dual mirror arrangement even with zero misalignment. The system cannot, however, vary scan radius conveniently without use of an additional wedge, as described in the next section. 


\subsection{Measured velocity and beam path in a tracking system with a two wedges}

For this case, expansion of equation (12) gives the final beam orientation $\hat{b}_{5}$ as:

$$
\begin{aligned}
\hat{b}_{5} & =\hat{x}\left[Q \sin \psi_{1} \sin \left(\Omega_{1} t+\varphi_{1}\right)+\sin \psi_{2}\left(R-S \frac{\varepsilon_{w 2}}{\varepsilon_{a}}\right) \sin \left(\Omega_{2} t+\varphi_{2}\right)\right] \\
& -\hat{y}\left[Q \sin \psi_{1} \cos \left(\Omega_{1} t+\varphi_{1}\right)+\sin \psi_{2}\left(R-S \frac{\varepsilon_{w 2}}{\varepsilon_{a}}\right) \cos \left(\Omega_{2} t+\varphi_{2}\right)\right] \\
& +\hat{z}\left[-\frac{\varepsilon_{w 1}}{\varepsilon_{a}}+Q \cos \psi_{1}+\cos \psi_{2}\left(R-S \frac{\varepsilon_{w 2}}{\varepsilon_{a}}\right)+T \frac{\varepsilon_{w 2}}{\varepsilon_{a}}-W\right]
\end{aligned}
$$

where:

$$
R=\left(\hat{b}_{3} \cdot \hat{u}_{H}\right)
$$

$S=\sqrt{1-\left(\frac{\varepsilon_{a}}{\varepsilon_{w 2}}\right)^{2}\left(1-\left(\hat{b}_{3} \cdot \hat{u}_{H}\right)^{2}\right)}$

$T=\left(\hat{b}_{4} \cdot \hat{u}_{J}\right)$

$$
W=\sqrt{1-\left(\frac{\varepsilon_{w 2}}{\varepsilon_{a}}\right)^{2}\left(1-\left(\hat{b}_{4} \cdot \hat{u}_{J}\right)^{2}\right)}
$$

Equation (24a) shows the complexity of the final beam orientation, even for this relatively simple optical arrangement, but it is easily obtained using the proposed procedure. By combination with equation (1), equation (24a) is used to derive the velocity sensed, following the same pattern as equations (22a-d). In the absence of target vibration, the measured velocity per unit rotation speed is given by: 


$$
\begin{aligned}
\frac{U_{m}}{\Omega_{1}} & =-y_{0} Q \sin \psi_{1} \sin \left(\Omega_{1} t+\varphi_{1}\right) \\
& -y_{0} \sin \psi_{2}\left[-Q \cos \psi_{1} \cos \psi_{2}+\frac{1}{\varepsilon_{a}}\left(\cos \psi_{2} \varepsilon_{w 1}-S \varepsilon_{w 2}\right)\right] \sin \left(\Omega_{2} t+\varphi_{2}\right) \\
& +y_{0} \sin \psi_{2}\left[Q \sin \psi_{1} \sin \psi_{2} \cos \left(\left(\Omega_{1} t+\varphi_{1}\right)-\left(\Omega_{2} t+\varphi_{2}\right)\right)\right] \sin \left(\Omega_{2} t+\varphi_{2}\right) \\
& -x_{0} Q \sin \psi_{1} \cos \left(\Omega_{1} t+\varphi_{1}\right) \\
& -x_{0} \sin \psi_{2}\left[-Q \cos \psi_{1} \cos \psi_{2}+\frac{1}{\varepsilon_{a}}\left(\cos \psi_{2} \varepsilon_{w 1}-S \varepsilon_{w 2}\right)\right] \cos \left(\Omega_{2} t+\varphi_{2}\right) \\
& +x_{0} \sin \psi_{2}\left[Q \sin \psi_{1} \sin \psi_{2} \cos \left(\left(\Omega_{1} t+\varphi_{1}\right)-\left(\Omega_{2} t+\varphi_{2}\right)\right)\right] \cos \left(\Omega_{2} t+\varphi_{2}\right)
\end{aligned}
$$

Equation (25) shows the influence of the rotation speeds of the wedges. By introducing differences in rotation speeds and initial angular positions and by adding angular oscillations, $\Omega_{B 1}$ and $\Omega_{B 2}$, to the rotation speeds, as shown in figure 4, different beam paths can be produced for real scanning applications. In all of the following simulations, the geometrical parameters used are: $\mathrm{z}_{\mathrm{B}}=0.5 \mathrm{~m}, \mathrm{z}_{\mathrm{G}}=$ $0.465 \mathrm{~m}, \overrightarrow{B C}|=| \overrightarrow{F G} \mid=5 \mathrm{~mm}$, wedge angles are $\psi_{1}=\psi_{2}=10$ degrees, refractive indices are $\varepsilon_{a}=1$ and $\varepsilon_{w 1}=\varepsilon_{w 2}=1.5$. In addition, there are no vibrations and all velocity components originate in the whole body rotation of the target.

The first case considers the wedges rotating at the same speed and in the same direction. The beam describes an apparently perfect circular path on the target, shown by the vector $\overrightarrow{O K}$ in figure $5 \mathrm{a}$, useful for tracking a rotating object when the wedge and target speeds are synchronised, $\Omega_{1}=\Omega_{2}=\Omega$. The size of the circle depends on the wedge angles, their relative initial angular positions and their zseparation. The velocity spectrum in figure $5 \mathrm{~b}$ does not show additional velocity components, an enhancement on that obtained with a dual mirror scanning 
arrangement where a $2 x$ scan frequency component is always detected.

If the wedges rotate in opposite directions, $\Omega_{1}=-\Omega_{2}$, the arrangement scans an almost straight line, as shown by the vector $\overrightarrow{O K}$ in figure $6 \mathrm{a}$ and $6 \mathrm{~b}$ where the orientation of the line depends on the initial phases of the wedges. The line width (between the out and back strokes of the scan) is directly influenced by the distance between the wedges. Thus, rotating the wedges at the same speed results in beam paths that can be used for general scanning and circular scanning applications. Rotating the wedges at different speeds, $\Omega_{2}=n \Omega_{1}$ where $n \neq \pm 1$, results in beam paths that are visually appealing, as exemplified by figures $7 \mathrm{a}$ and 7b, but without an obvious application in scanning LDV.

The introduction of anti-phase oscillations at frequencies $\Omega_{B 1}$ and $\Omega_{B 2}$ on top of equal whole body rotations, see figure 4 , gives opportunity to track and scan simultaneously as has been useful previously for application on bladed disks [11, 13]. When the oscillation frequency of the wedges is higher than the rotation frequency, for example $\Omega_{1}=\Omega_{2}=\Omega$ and $\Omega_{B 2}=-\Omega_{B 1}=4 \Omega$, the result is a fast scan up and down the blade, several times within each rotation, as described by the vector $\overrightarrow{O K}$ in figure 8 a. Similar scan paths have been created previously, by simulation [12] and experimentally using the dual mirror arrangement [13] to scan a beam up and down a blade several times during each rotation cycle. With this arrangement, time-varying components of the whole body rotation velocity of the target will be detected and the spectrum shown in figure $8 \mathrm{~b}$ reveals additional 
terms at DC and integer multiples of the oscillation frequency. These undesired peaks may adversely affect the intended vibration measurements because they may coincide with frequencies of interest. An oscillation frequency smaller than the rotation frequency may be more appropriate, resulting in a slow scan up and down the blades over several rotations, as shown by the vector $\overrightarrow{O K}$ in figure 9a, $\left(\Omega_{1}=\Omega_{2}=\Omega\right.$ and $\left.\Omega_{B 2}=-\Omega_{B 1}=0.1 \Omega\right)$. The spectrum again reveals undesired components at DC and integer multiples of the oscillation frequency but these are now well below the frequencies likely to be of interest. In either case, the amplitudes of the harmonics are of similar magnitude and the lower multiples may be of measurable amplitude in a real application.

These results show the potential of the two wedge arrangement for scanning applications, including use on rotating targets. The data also show the merit of the proposed procedure for predicting paths for complex configurations and for identifying any undesired measured velocities resulting from the whole body target rotation. This assists the user in ensuring correct interpretation of measured data.

\subsection{Initial experimental results}

Initial experiments have been performed to begin to confirm the mathematical model introduced in this paper. The test rig incorporates two rotating wedges, positioned as in figure 2. An encoder positioned behind the rotating target drives a signal generator which controls the rotation of the wedges using stepper motors 
and belt drives. Rotating the wedges in the same direction and at the same speed, a circular scan profile with radius of $5 \mathrm{~cm}$ was created at $10 \mathrm{~Hz}$. In the real experiment, it is obviously not possible to set up the system with zero misalignment. This is particularly the case as, at this stage of the research, no systematic alignment procedure yet exists for this novel configuration. By mounting the wedges on simple translation stages, a basic alignment was performed and figure 10 reveals velocity components of $1.46 \mathrm{~mm} / \mathrm{s}$ and of $0.086 \mathrm{~mm} / \mathrm{s}$ respectively at $1 \mathrm{x}$ and $2 \mathrm{x}$ target rotation speed. These components are due to misalignment and the incorporation of misalignments into the model presented in this paper is the subject of the next phase of this project. For now, it is noteworthy that the sensitivity to misalignment compares very favourably with the conventional dual mirror system in which residual misalignments, even after completion of the dedicated alignment procedure, might result in a measured velocity at $1 \mathrm{x}$ scan frequency approximately ten times higher while the mirror configuration itself would result in a $2 x$ scan frequency component approximately fifty times higher.

\section{Conclusions}

Scanning Laser Doppler Vibrometers require laser beam manipulation by optical devices along the beam path. This paper has proposed a general procedure able to determine the final beam orientation and a known point along the beam for arrangements in which a beam is refracted and/or reflected. Combining use of rotation matrices with vector expressions for refraction and reflection, the final 
incident beam direction can be determined for any kind of scanning arrangement. The procedure has been applied for the first time to novel scanning LDV arrangements in which one or two rotating wedges enable the tracking of a rotating component, avoiding the problems of inertia encountered in galvanometer mirrorbased systems. The procedure has shown that the wedge angles, their separation and their relative initial angular position determine the radius of the circular tracking paths. Knowledge of final beam orientation and known point has been used to predict measured velocity in these tracking applications. In the absence of misalignment, the wedge-based circular tracking systems do not show any undesired velocity components from the target rotation, offering a significant advantage over the conventional dual mirror scanning system which suffers from the presence of an unavoidable velocity component at twice scan frequency. Furthermore, the addition of anti-phase angular oscillations to the wedge rotation speeds can provide a scan up and down the target while tracking. Even for this more complex arrangement, measured velocities have been readily predicted.

Initial experimental measurements performed with two rotating wedges have been encouraging. Undesired velocity components related to inevitable misalignments were detected at $1 \mathrm{x}$ and $2 \mathrm{x}$ scan frequency rotation speed but these were much lower than those obtained with the dual mirror scanning system, especially at $2 \mathrm{x}$. Further work will incorporate misalignments into the predictions, together with Doppler shifts occurring on transmission through the wedges themselves. 


\section{Appendix 1}

The surface normal directions for the wedges are obtained using rotation matrices.

For example, for a single wedge arrangement, the normal $\hat{u}_{c}$ for the inclined surface of the wedge is defined as:

$\hat{u}_{C}=\left[\begin{array}{lll}\hat{x} & \hat{y} & \hat{z}\end{array} \llbracket Z, \gamma_{1}\right] \llbracket X, \psi_{1} \rrbracket\left[\begin{array}{lll}0 & 0 & -1\end{array}\right]^{T}$

where the matrices $\left[Z, \gamma_{1}\right]$ and $\left[X, \psi_{1}\right]$ represent anticlockwise rotations around the $x$ and the $z$ axes and are defined as [14] :

$$
\left[Z, \gamma_{1}\right]=\left[\begin{array}{ccc}
\cos \gamma_{1} & -\sin \gamma_{1} & 0 \\
\sin \gamma_{1} & \cos \gamma_{1} & 0 \\
0 & 0 & 1
\end{array}\right] \quad\left[X, \psi_{1}\right]=\left[\begin{array}{ccc}
1 & 0 & 0 \\
0 & \cos \psi_{1} & -\sin \psi_{1} \\
0 & \sin \psi_{1} & \cos \psi_{1}
\end{array}\right]
$$

Other uses of rotation matrices in this paper follow the same format.

\section{References}

[1] Davis Q. V. and Kulczyk W. K. Vibrations of turbine blades by means of a laser. Nature 1969; 222, pp 475-76.

[2] Hancox J., Staples B. C. and Parker R. J. The application of scanning laser

Doppler Vibrometry in aero-engine development. Proc.Inst. Mech. Eng. 1995; 209: 35-42. 
[3] Stanbridge A. B. and Ewins D. J. Modal testing using a scanning laser Doppler vibrometer. Mech. Syst. Signal Process 1999; 13: 255-270.

[4] Castellini P. and Paone N. Development of the tracking laser vibrometer: performance and uncertainty analysis. Rev. Sci. Instrum. 2000; 71: 4639-47.

[5] Boedecker S., Dräbenstedt A., Heller L., Kraft A., Leonhardt A., Rembe C. Optical Derotator for Scanning Vibrometer Measurements on Rotating Objects. Seventh International Conference on Vibration Measurements by Laser Techniques: Advances and Applications, Proc. of SPIE Vol. 6345, Ancona, Italy, 2006.

[6] Lomenzo R. A., Barker A. J., Wicks A. L. and King P. S. A Laser Vibrometry System for Measuring Vibrations on Rotating Disks. $4^{\text {th }}$ National Turbine Engine High Cycle Fatigue (HCF) Conference, Monterey, CA, 1999; 277-282.

[7] Sever I.A. Turbomachinery Blade Vibration Measurements with Tracking LDV under rotation. Seventh International Conference on Vibration Measurements by Laser Techniques: Advances and Applications, Proc. of SPIE Vol. 6345 Ancona, Italy, 2006.

[8] Halkon B.J. and Rothberg S.J. Vibration measurements using continuous scanning laser vibrometry: theoretical velocity sensitivity analysis with applications. Meas. Sci.Technol. 2003; 14: 382-389.

[9] Bell J.R., Rothberg S.J. Laser vibrometers and contacting transducers, target rotation and 6 degree-of-freedom vibration: what do we really measure? Journal of 
Sound and Vibration 2000; 237 (2): 245-261.

[10] Amirault C. T. and DiMarzio C. A. Precision pointing using a dual-wedge scanner. Applied Optics, 1985; Vol. 24, No. 9, 1 May: 1302-1308.

[11] Di Maio D. and Ewins D. J. CAISER MYMESIS, a new software platform for Virtual and Actual Vibration Testing on Rotating Structures using a Continuously Scanning LDV Technique. Proceedings of Seventh International Conference on Vibration Measurements by Laser Techniques, 5503, Proc. of SPIE Vol. 6345, 63450Q, Ancona, Italy, 2006.

[12] Gerald F. Marshall. Risley Prism Scan Patterns. Part of the SPIE Conference on Optical Scanning: Design and Application, Denver, Colorado, July 1999, SPIE Vol. 3787: 74-86.

[13] Halkon B. J. and S. J. Rothberg. Synchronised-Scanning Laser Vibrometry. Proceedings of Sixth International Conference on Vibration Measurements by Laser Techniques, 5503, Ancona, Italy, 2004: 260-271, ISSN 0277-786X.

[14] Harrison H.R. and T.Nettleton. Advanced Engineering Dynamics, Arnold Edition, 1997. 


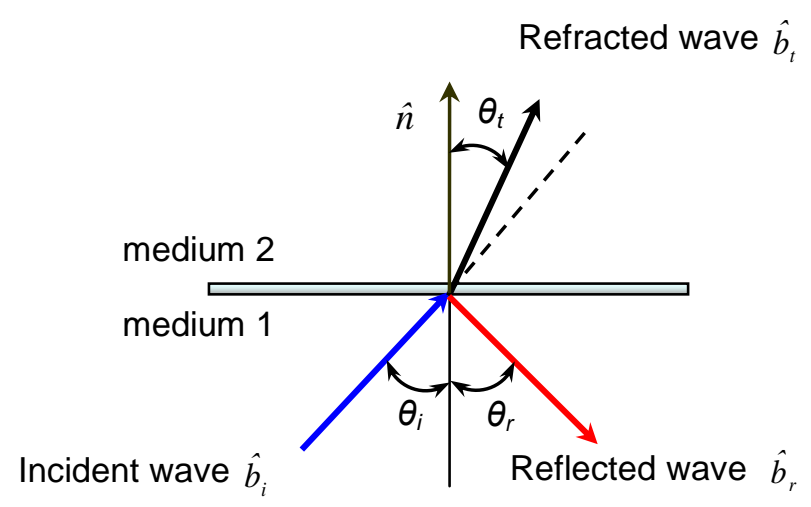

Figure 1 - Reflection and refraction showing unit vectors

Rotating target

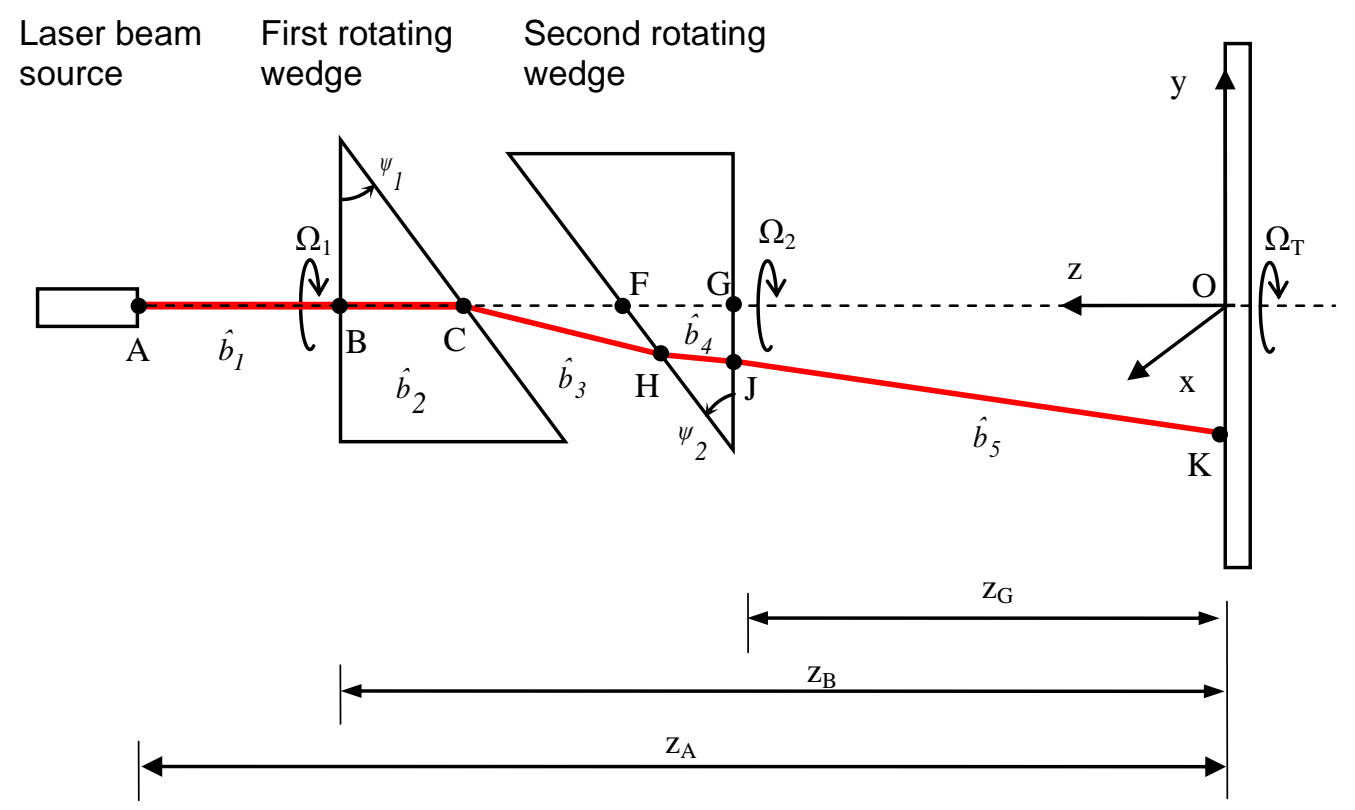

Figure 2 -Tracking system with two rotating wedges 

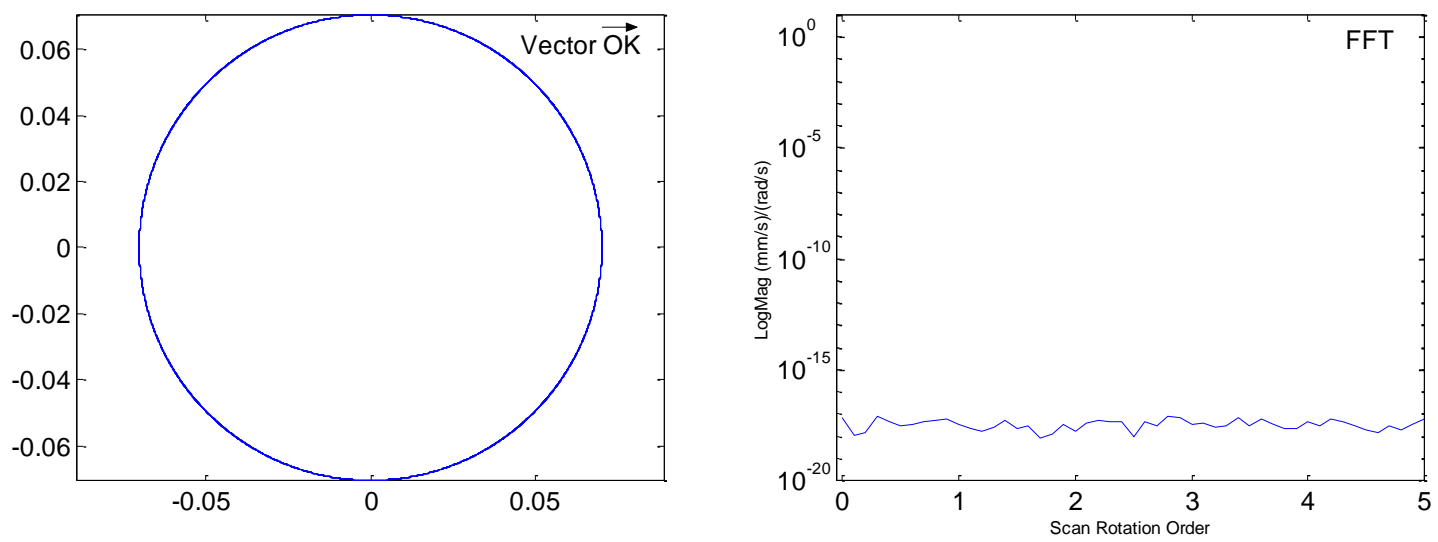

Figure 3 - Simulation of scanning system with a single rotating wedge: a) Beam path $b$ ) velocity spectrum

$$
\mathrm{z}_{\mathrm{B}}=0.5 \mathrm{~m} \text { and } \psi_{1}=10 \text { degrees }
$$

$\Omega_{B I}=$ Oscillation frequency on the top of the first body wedge

$\Omega_{B 2}=$ Oscillation frequency on the top of the second body wedge

$\Omega_{1}=$ Rotational speed of the first wedge

$\Omega_{2}=$ Rotational speed of the second wedge

$\Omega \quad=$ Rotational speed of the target

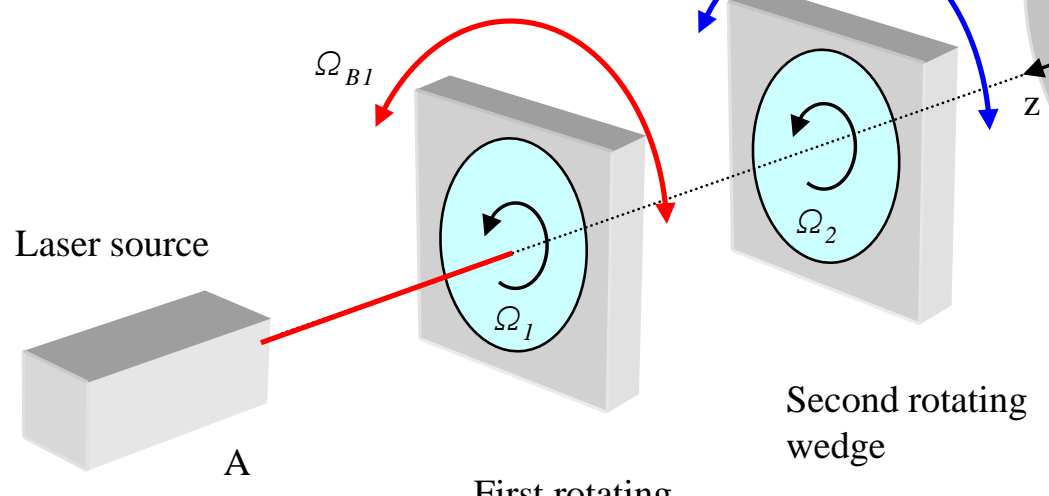

First rotating wedge

Figure 4 - Schematic representation of all the rotations and oscillations considered for the double wedge scanning arrangement 

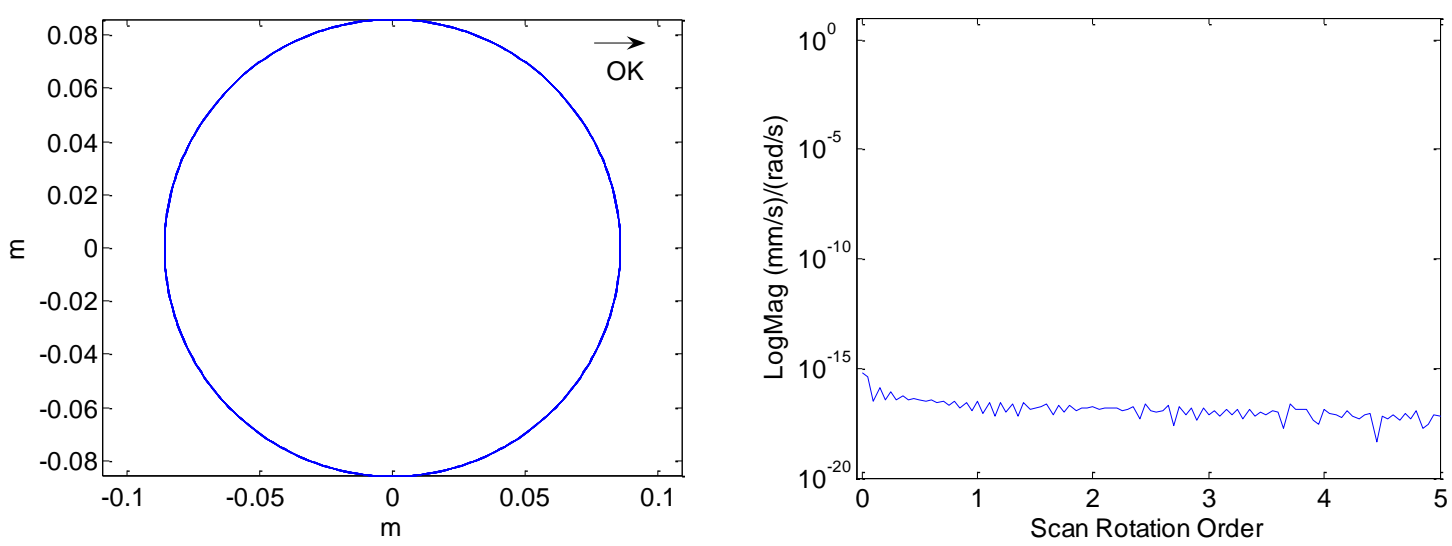

Figure 5 - Simulation of scanning system with two wedges rotating at the same speed: a) Beam path b) velocity spectrum. Geometrical parameters: $\mathrm{z}_{\mathrm{B}}=0.5 \mathrm{~m}, \mathrm{z}_{\mathrm{G}}=0.465 \mathrm{~m}, \psi_{1}=\psi_{2}=10$ degrees, $\varphi_{2}-\varphi_{1}=180^{\circ}$
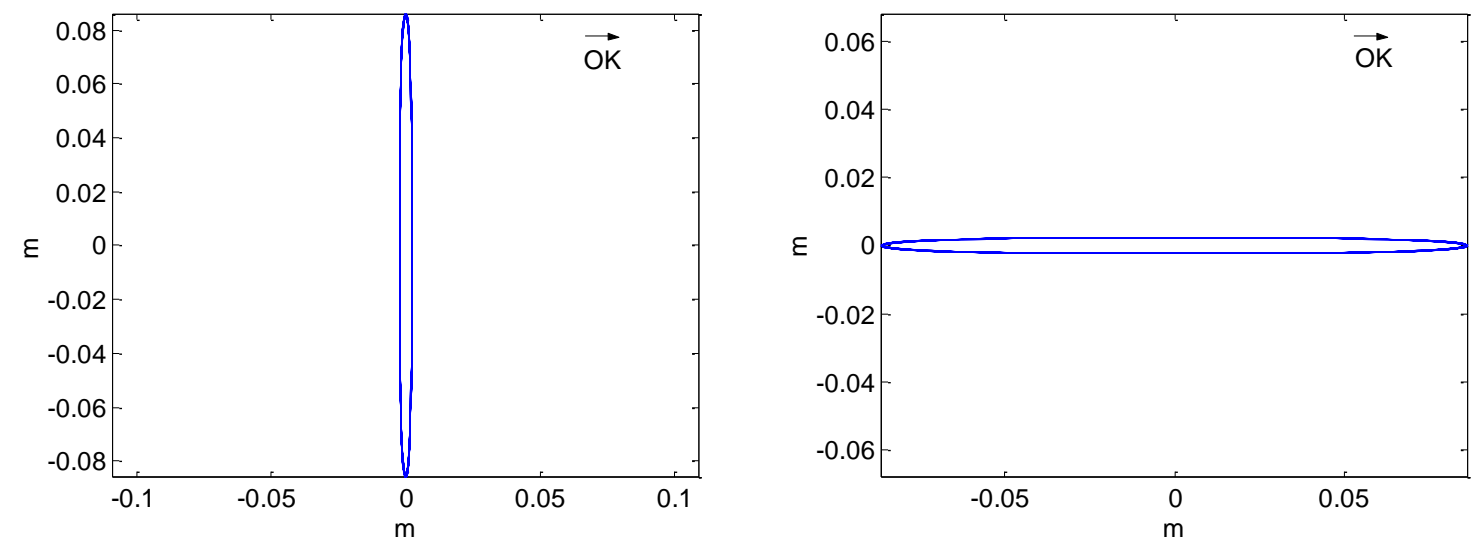

Figure 6 - Simulation of beam path for two wedges rotating in opposite directions at the same speed: a) $\varphi_{2}-\varphi_{1}=180^{\circ}$ b) $\varphi_{2}-\varphi_{1}=0^{\circ}$. Geometrical parameters: $z_{B}=0.5 \mathrm{~m}, z_{G}=0.465 \mathrm{~m}, \psi_{1}=\psi_{2}=10$ degrees 

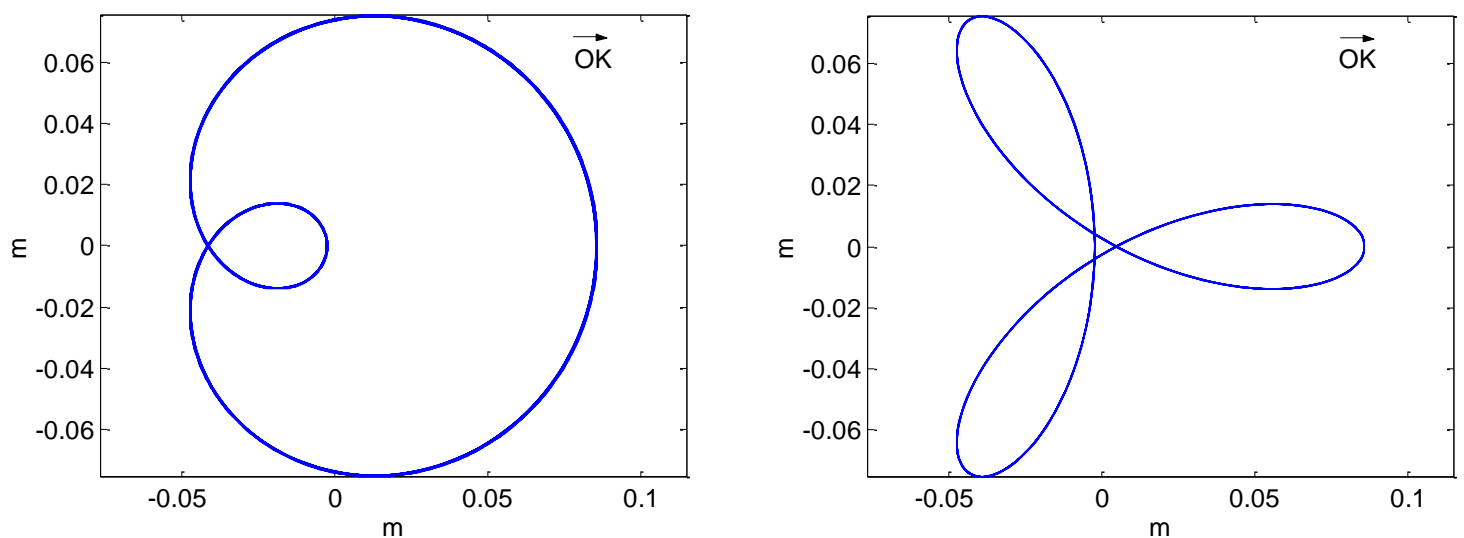

Figure 7 -Simulation of beam paths for two wedges rotating at the different speeds: a) $\Omega_{2}=2 \Omega_{1}$, b) $\Omega_{2}=-2 \Omega_{1}$ Geometrical parameters: $\mathrm{z}_{\mathrm{B}}=0.5 \mathrm{~m}, \mathrm{z}_{\mathrm{G}}=0.465 \mathrm{~m}, \psi_{1}=\psi_{2}=10$ degrees $\varphi_{2}-\varphi_{1}=90^{\circ}$ 

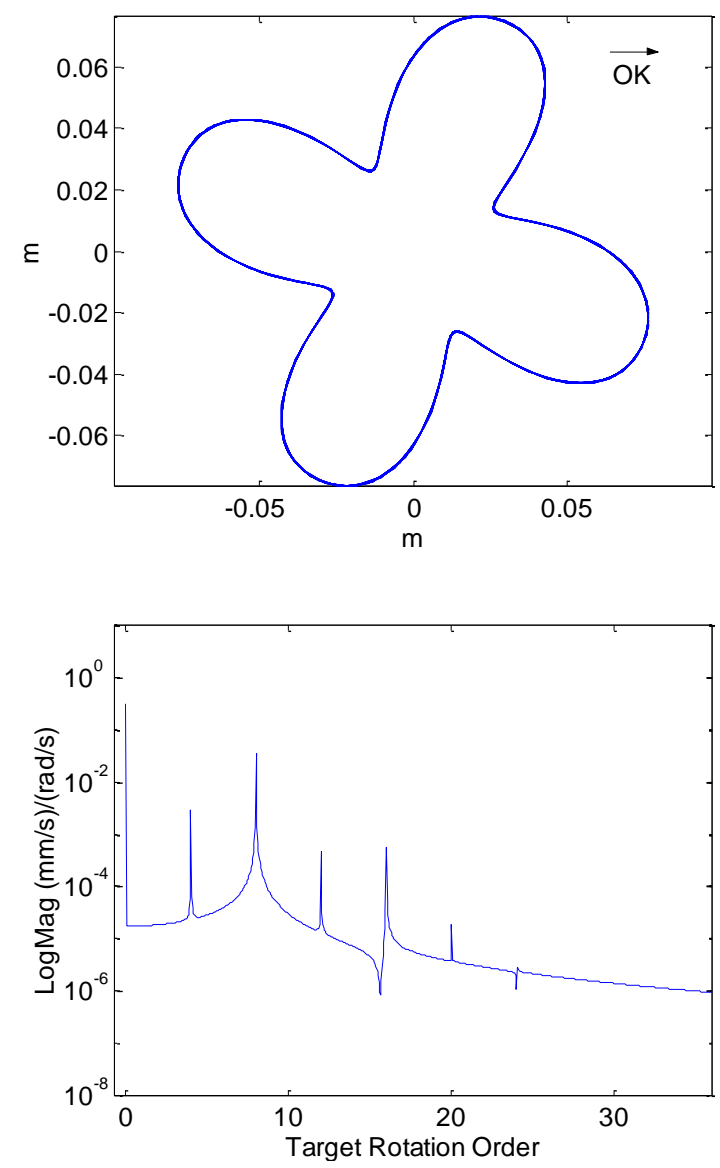

Figure 8 - Simulation of scanning system with two wedges rotating at the same mean speed with superimposed anti-phase oscillations at $4 \mathrm{x}$ rotation frequency: a) beam path, b) velocity spectrum. Geometrical parameters: $\mathrm{z}_{\mathrm{B}}=0.5 \mathrm{~m}, \mathrm{z}_{\mathrm{G}}=0.465 \mathrm{~m}, \psi_{1}=\psi_{2}=10$ degrees, $\varphi_{2}-\varphi_{1}=90^{\circ}$ 

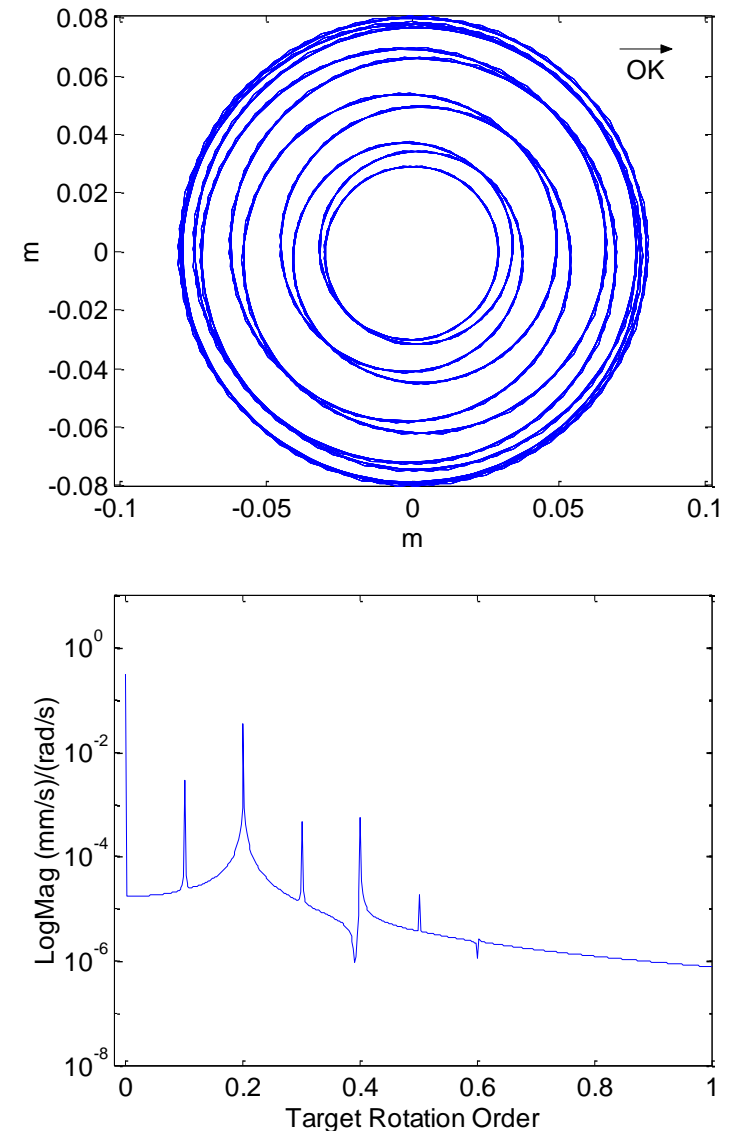

Figure 9 - Simulation of scanning system with two wedges rotating at the same mean speed with superimposed anti-phase oscillations at $0.1 \mathrm{x}$ rotation frequency: a) beam path, $\mathrm{b}$ ) velocity spectrum. Geometrical parameters: $\mathrm{z}_{\mathrm{B}}=0.5 \mathrm{~m}, \mathrm{z}_{\mathrm{G}}=0.465 \mathrm{~m}, \psi_{1}=\psi_{2}=10$ degrees, $\varphi_{2}-\varphi_{1}=90^{\circ}$ 


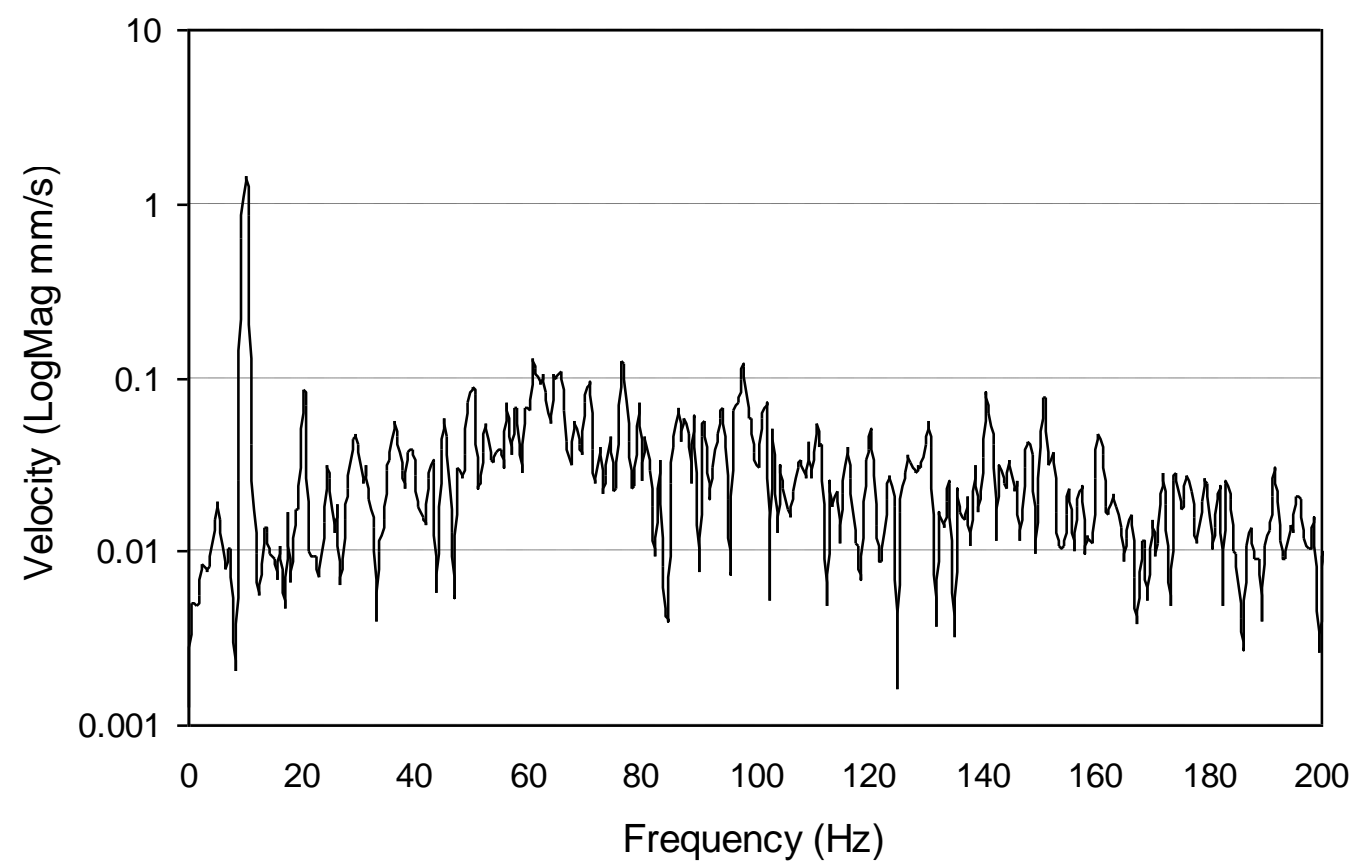

Figure 10 - Experimental data for tracking system (two wedges rotating at the same mean speed) 